FERMILAB-TM-2068

\title{
Comparison of Fiber Polishing Techniques
}

P. Hanlet, M. Marcus and E. Gallas

Fermi National Accelerator Laboratory

P.O. Box 500, Batavia, Illinois 60510

March 1999 


\section{Disclaimer}

This report was prepared as an account of work sponsored by an agency of the United States Government. Neither the United States Government nor any agency thereof, nor any of their employees, makes any warranty, expressed or implied, or assumes any legal liability or responsibility for the accuracy, completeness, or usefulness of any information, apparatus, product, or process disclosed, or represents that its use would not infringe privately owned rights. Reference herein to any specific commercial product, process, or service by trade name, trademark, manufacturer, or otherwise, does not necessarily constitute or imply its endorsement, recommendation, or favoring by the United States Government or any agency thereof. The views and opinions of authors expressed herein do not necessarily state or reflect those of the United States Government or any agency thereof.

\section{Distribution}

Approved for public release; further dissemination unlimited.

\section{Copyright Notification}

This manuscript has been authored by Universities Research Association, Inc. under contract No. DE-AC02-76CHO3000 with the U.S. Department of Energy. The United States Government and the publisher, by accepting the article for publication, acknowledges that the United States Government retains a nonexclusive, paid-up, irrevocable, worldwide license to publish or reproduce the published form of this manuscript, or allow others to do so, for United States Government Purposes. 
DØ Note 3561

January 28, 1999

\title{
Comparison of Fiber Polishing Techniques
}

\author{
Pierrick Hanlet, Matthew Marcus \\ Northeastern University \\ Elizabeth Gallas \\ University of Texas at Arlington
}

Many DØ Run II detectors currently in production rely on scintillating tile and fiber technology. In general, light from active scintillating elements or calibration signals is transported to the photodetection system along optical fiber pathways. Building a tile/fiber detector requires very conscientious technical support and a high degree of quality control; polishing fibers is one of the most delicate of tasks involved.

This note compares three methods used to polish Hewlett Packard HFBR-RUS500 fiber. This type of fiber is expected to be used in both the Muon Scintillator Counters (MSC) and the InterCryostat Detector (ICD) calibration systems to transport light from the LED distribution block to the photomultiplier tubes.

\section{Contents}

1 Introduction $\quad 2$

2 Polishing Techniques 2

2.1 Teflon Block Method . . . . . . . . . . . . . . . . . 2

2.2 Ice Polishing Method . . . . . . . . . . . . . . . . . . 3

2.3 The Fiberfin III . . . . . . . . . . . . . . . . . . . . . 3

3 Polishing Results $\quad 3$

4 Transmission Results $\quad 5$

5 Single Fiber Polishing with Connectors $\quad 6$

$\begin{array}{llr}6 & \text { Conclusions } & 7\end{array}$

$\begin{array}{llr}7 & \text { Acknowledgments } & 7\end{array}$ 


\section{Introduction}

Both the MSC[1, 2] and ICD[3] scintillator groups expect to use Hewlett Packard (HP) HFBRRUS500 fiber[4] to transport light from the Light Mixing Boxes (LMBs)[5] to the photomultiplier tubes (PMTs) for their calibration systems. This HP fiber is a single clad $1.0 \mathrm{~mm}$ diameter clear plastic fiber wrapped in a $2.2 \mathrm{~mm}$ diameter black polyethylene jacket. The black jacket protects the fiber and also serves to optically isolate each fiber from other fibers as well as from external light sources. Although the fiber is singly clad, light transmission loss is relatively low, $0.22 \mathrm{~dB} / \mathrm{m}$, such that light loss is not a significant concern for our fiber lengths of less than $7 \mathrm{~m}$.

Three fiber polishing methods were considered in this study and are briefly described in the next section. The first two methods, the "teflon block" and "ice polishing" methods, polish numerous fibers simultaneously; the names of the methods refer to the means by which the fibers are held for polishing ${ }^{1}$. The third method is a single fiber polisher. The reason for investigating this technique when multiple fiber polishing would obviously be faster, is that it allows one to polish the fiber after an optical connector is installed on the fiber. This is highly desirable since installation of the connector can damage the polished surface if the fiber is mishandled, and because the crimping of the connector onto the fiber causes the fiber to retract within the connector. The retraction of the fiber yields poorer quality connections of the two mating fibers in a bulkhead connector since the fibers are not flush with the ends of the connectors. In addition, the less than 3 second polishing time per fiber does not significantly slow down the process of polishing fibers, since one no longer needs to load a fixture (teflon or ice), or wait for the water to freeze or thaw (ice).

Each method was used to polish 5 pieces of HP fiber without connectors; the results are described in Sec. 2. Sec. 5 will describe the polishing tests of the single fiber polisher with the connectors installed.

\section{Polishing Techniques}

\subsection{Teflon Block Method}

This and the following ice polishing method are described in detail in DØ note 3390 [6]. Both methods use a diamond flywheel cutting machine, the $P$ 3, which has a rotating wheel with progressively finer cutting bits at decreasing radii: one or more course cutting carbide blades at outer radial points, a course diamond bit at an intermediate point and a fine cutting diamond bit at the innermost radii. In a single pass, the object to be polished moves at a constant speed along a linear path intercepting the bits on the spinning wheel along an inward radial path. By pushing a single (START) button, the machine is automated to start the rotating wheel, move the piece smoothly with precise positioning into the cutting bits and return the piece to the starting position.

For this polishing procedure, fibers are inserted into holes of a teflon block. Fibers should fit snugly in the holes so that the cladding and jacket are held fixed during polishing. The fibers are polished by facing off the end of the block with one or more passes with a diamond flywheel polishing machine.

\footnotetext{
${ }^{1}$ It is worth noting that though the teflon block method is a multiple fiber polisher, it typically polishes an order of magnitude fewer fibers than does the ice polishing method.
} 


\section{$2.2 \quad$ Ice Polishing Method}

This method is fully described in DØ note 3390 [6] and like the Teflon block method, uses a diamond flywheel cutting machine. Briefly, hundreds of fibers are packed into the end of a fixture which is subsequently filled with water and frozen in liquid nitrogen. The end of the frozen fixture is then faced off with the $P 3$ diamond flywheel polishing machine located at Fermilab in Lab 7.

Previous experience has shown this technique to yield high quality polishes for many types and sizes of fibers. Another advantage of this technique is that hundreds of fibers can be polished in a comparatively short period of time compared to any single fiber polishing technique.

\subsection{The Fiberfin III}

The Fiberfin III is a new machine and shows great promise. Previous versions of this machine (described in DØ note 3390) were too labor intensive to be considered for this application. This new machine was developed and designed by Carl Lindenmeyer and others in the Fermilab Particle Physics Division Technical Centers. The unit used here was commercially produced by the PM Manufacturing Service, Inc [7].

The Fiberfin III is lightweight and portable, featuring simple one lever operation with a completed polishing cycle in less than 3 seconds. Rather than using a small diamond which rotates incrementally across the surface to be polished, this polisher chops away smaller and smaller slices of the fiber along the fiber axis. The diamond in this machine has a smooth edge about $1 / 8^{\prime \prime}$ wide. A collet grips the end of the fiber to be polished. For the studies performed here, a custom collet holds the $2.2 \mathrm{~mm} \mathrm{HP}$ fiber and jacket. As long as the diamond remains intact, the machine yields smooth polishes.

\section{Polishing Results}

Each method was used to polish 5 pieces of HP HFBR-RUS500 fiber. The results of the three polishing techniques are shown in Figs. 1-3. Each photograph is typical of all fibers polished with that technique. In the left side photographs, the fiber surfaces being studied are illuminated; in the right side photographs, the fibers are illuminated from the opposite ends of the fibers. Smearing of the black polyethylene onto the clear fiber core is not observed for any of the techniques demonstrated here.

Shown in Figure 1 is a picture of the end of a fiber polished using the teflon block technique. While cladding damage was not explicitly a problem, a smooth polish across the fiber face is not observed. It is suspected that the teflon block aperture is unable to hold the end of the fiber firmly enough during the polishing cycle yielding an unsatisfactory finish for all trials.

Figure 2 shows the end of a fiber polished using the ice polishing technique. Smooth polishes are observed in all cases, with striation (parallel lines) on the fiber and the casing due to the cutting method of the P3 polisher. The striation observed is not expected to significantly effect light transmission, so this method meets the polishing quality criteria.

Figure 3 shows the end of a representative fiber polished using the Fiberfin III single fiber polishing machine. Smooth polishes are observed in all 5 trials. On several of the fibers, a crescent shaped deformation was observed at the edge of the fiber with this cutting method; the effect is small and is not expected to significantly affect the fiber's light transmission properties. This method is judged to be superior to the teflon block technique for polishing 

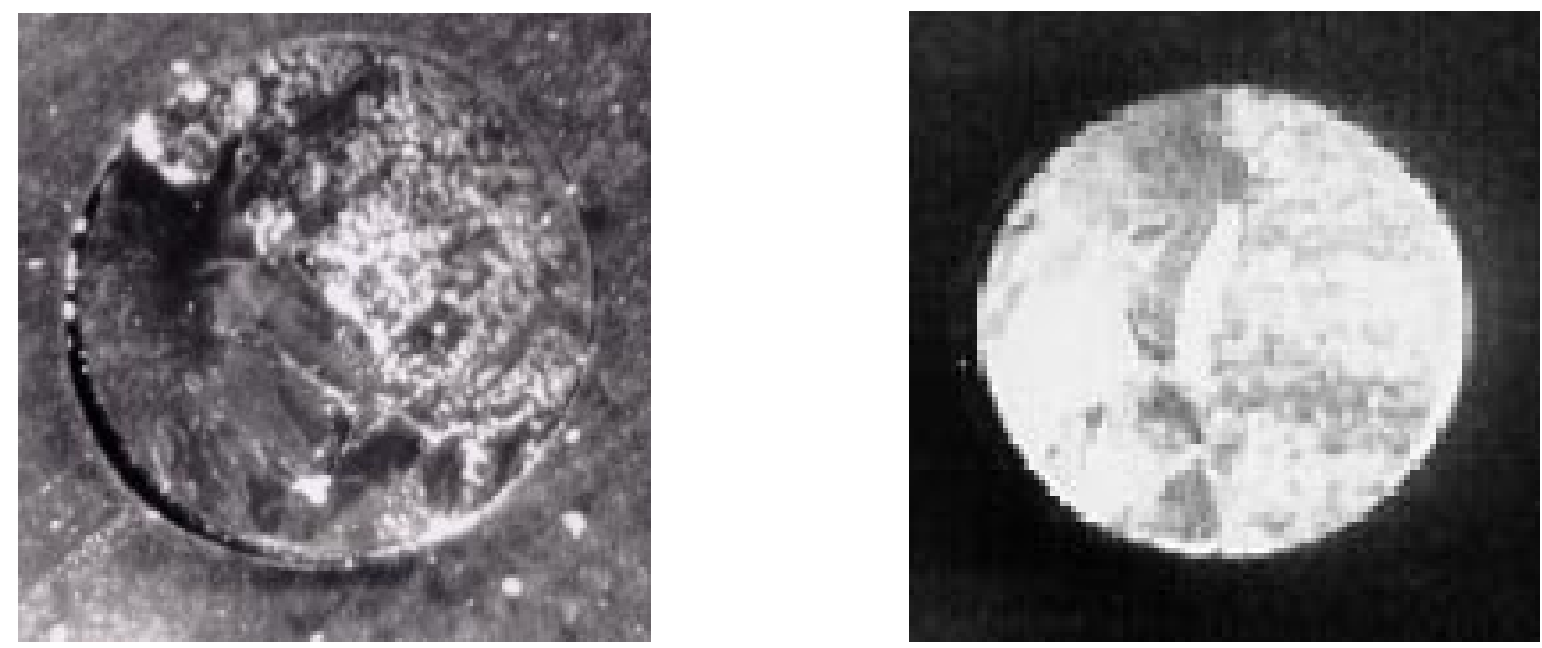

Figure 1: Fiber end polished using the teflon block technique.
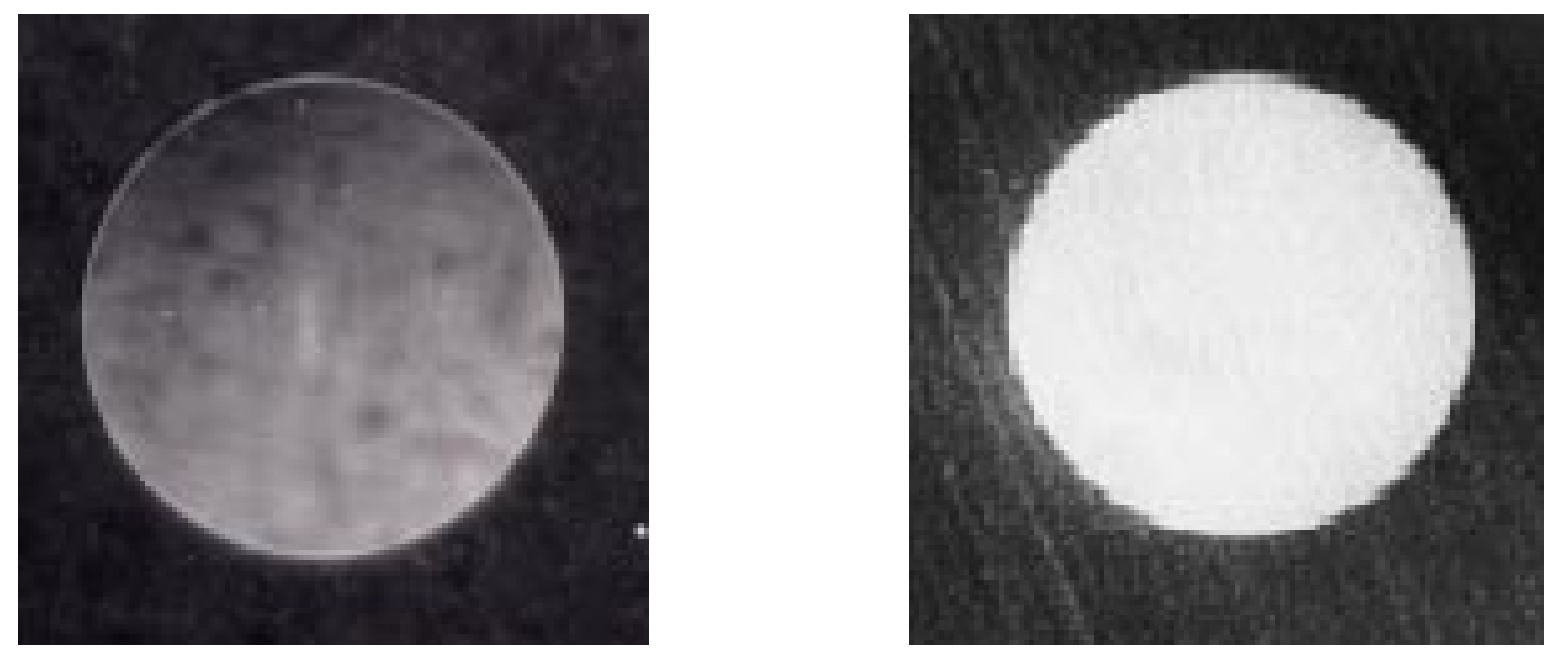

Figure 2: Fiber end polished using the ice polishing technique.
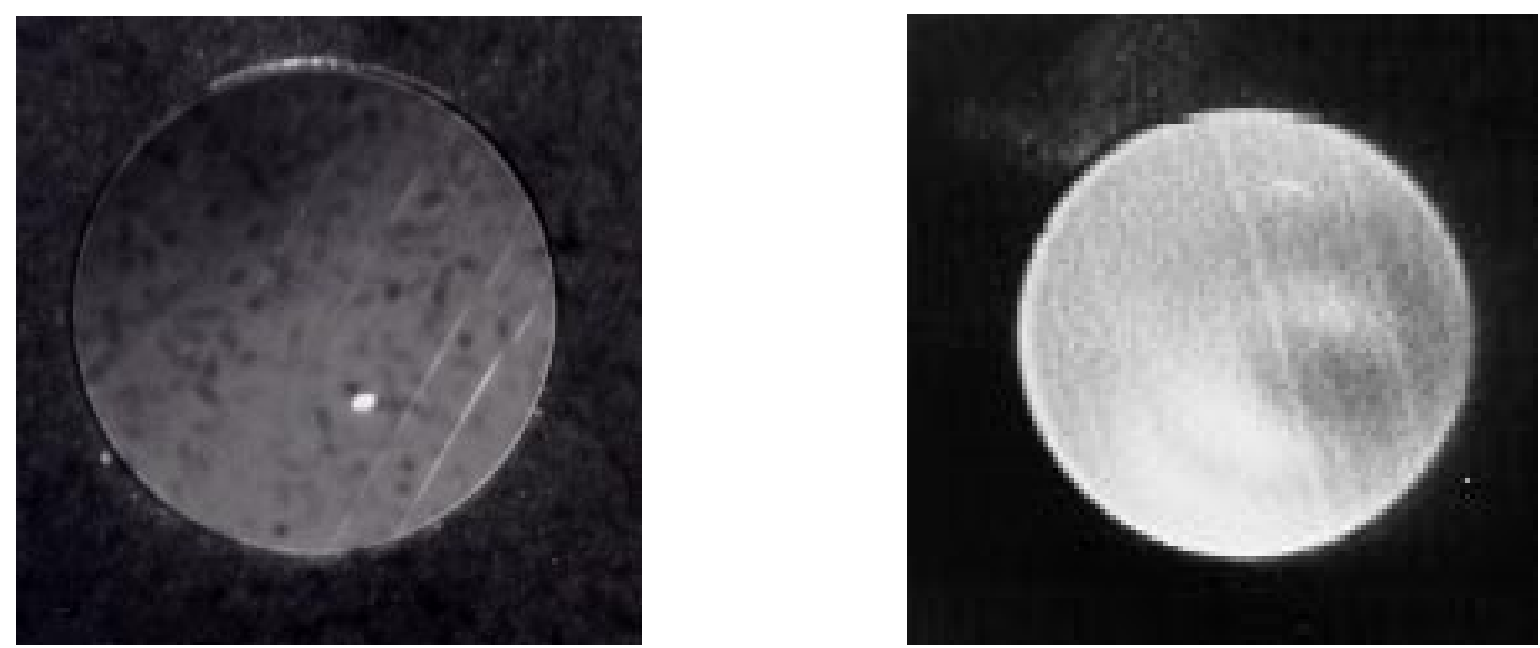

Figure 3: Fiber end polished using the Fiberfin III single fiber polishing machine, manufactured by PM Manufacturing Service, Inc. 
quality and requires less time to actually execute the polish, when considering setup time. This method meets the quality criteria for a smooth polish.

\section{Transmission Results}

The acid test in comparing the various polishing techniques is the measurement of light transmission through the polished surface of the fiber. To make this determination, we illuminated each fiber with a pulsed LED and measured the light transmitted through the fiber into a PMT.

To minimize the systematic uncertainties in the measurements due to inhomogeneities in the LED surface and PMT photocathode, a single fiber was glued to the LED, and another to the PMT. The LED fiber ensures that the same amount of light illuminates the fiber to be tested. The PMT fiber ensures that the light going to the PMT's photocathode is met with the same quantum efficiency for each fiber tested. Over the less than 2 hour time span of the tests, both LED and PMT were known to be stable to within $1 \%$.

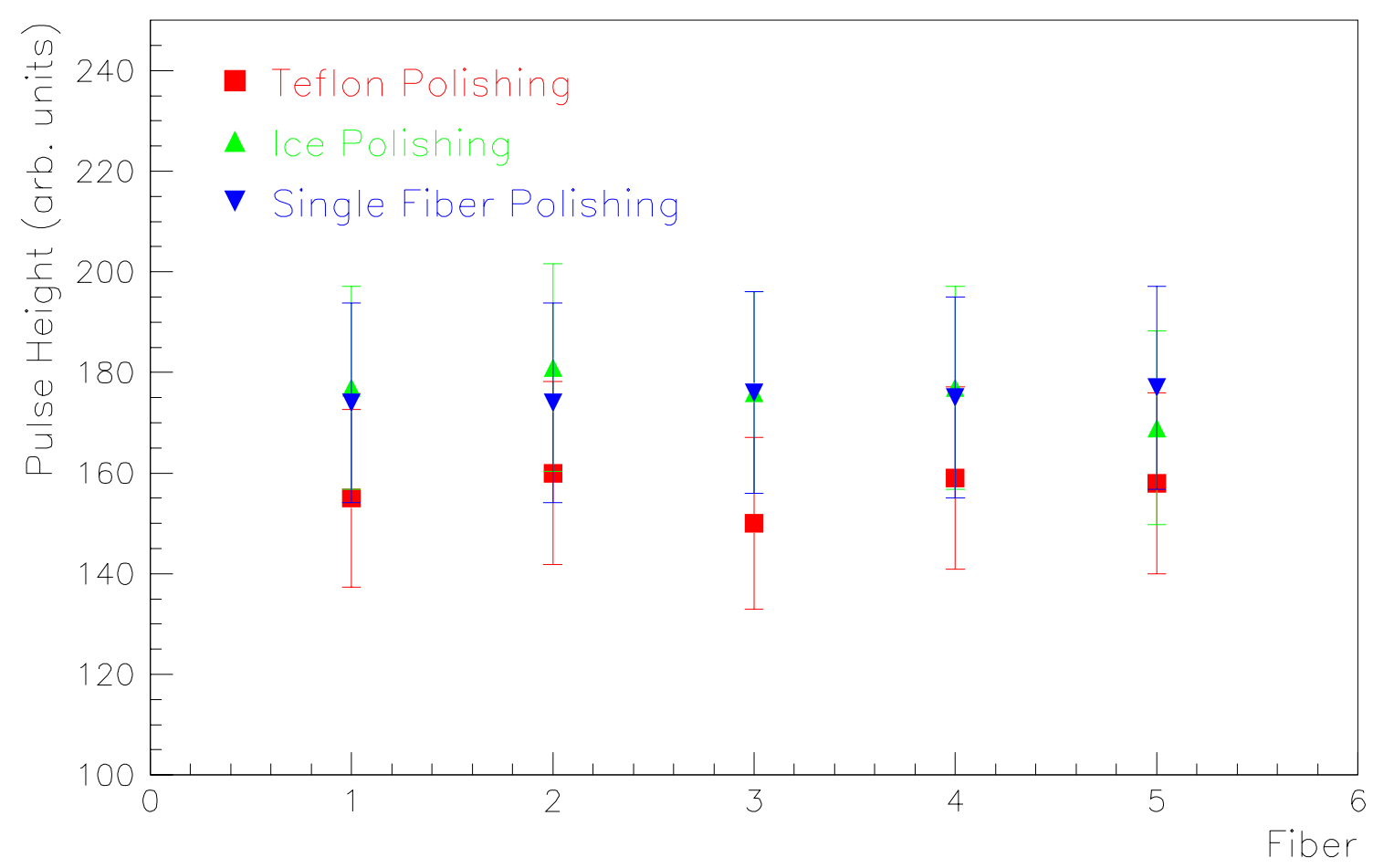

Figure 4: Comparison of the light transmission through 15 fibers.

In addition, all 15 fibers to be tested fibers were cut to the same length and were polished at one end with the Fiberfin III single fiber polisher; the other ends of the fibers remained polished using the three techniques described in Sec. 2. The LED fiber end was inserted halfway into a $3.6 \mathrm{~cm}$ lucite block with a $2.2 \mathrm{~mm}$ through hole; the PMT fiber was inserted halfway into another $3.6 \mathrm{~cm}$ lucite block with a $2.2 \mathrm{~mm}$ through hole. The fibers to be tested were then held in between and aligned with the LED fiber and the PMT fiber by inserting each end into the other ends of the two lucite block holes. The PMT output was integrated using a LeCroy $3001 \mathrm{qVt}$ in charge mode, and the peak of the distribution was recorded. 
The light transmission results for each of the 15 fibers are shown in Fig. 4. The 11\% error was determined by reinserting the fiber and repeating the measurement with a single fiber 20 times and computing the RMS. Though the errors are large, the measured means clearly indicate $\mathrm{a} \sim 10 \%$ decrease in light transmission through fibers polished with the teflon block technique. The ice and single fiber polishing techniques yield consistently higher light transmission and are essentially indistinguishable. Given the magnitude of the errors, one cannot observe any effect of the fiber deformation with the single fiber polisher or the striations of the ice polishing.

\section{$5 \quad$ Single Fiber Polishing with Connectors}

Given the successful results of the Fiberfin III, we were driven to assure that the application of a connector would not deteriorate the results. To polish the fibers with a connector, the Fiberfin III was modified to use a custom collet which holds the HP simplex connectors.


Figure 5: Fiber with connector: left—polished without using glue, right-polished using glue to stabilize the fiber within the connector and right.

One of the concerns was that the fiber ends would vibrate within the connector when polishing. To help prevent this, we dipped the end of the fibers in glue before inserting them into the connectors. When comparing the results with and without glue, we found that $75 \%$ of the fibers failed without glue and $20 \%$ failed with glue. The failure in these cases was a gouge in the polished fiber. A comparison of fibers polished in the connectors can be seen in Fig. 5, where the gouge can be seen in the left hand photograph.

Further investigation led to the determination that the gouging was due in large part to the length of protruding fiber from the connector before polishing. When tested with glue, 20 fibers each had gouging when the fiber protruded by more than $2 \mathrm{~mm}$, and none when the fibers were stripped leaving a protrusion of less than $2 \mathrm{~mm}$. 


\section{Conclusions}

We conclude that two of the three fiber polishing techniques evaluated yields polished fibers meeting the quality control criteria, namely the ice polishing and Fiberfin III polishing techniques.

The MSC LED monitoring system design calls for the ends of fibers to be inserted into HP connectors which plug into a standard HP bulkhead connector. In order to maintain a uniform distance at the connector, it is desirable to polish these fibers within the connector. Because the ice polishing method does not allow for a connector of this type inside the polishing fixture, Fiberfin III single fiber polishing method will be employed using a custom collet that fits over a fiber simplex HP connector.

If the ICD group uses this type of fiber in their LED calibration system, the ice polishing technique will be used because no such connector is required.

\section{Acknowledgments}

The authors would like to thanks the following people for their assistance in evaluating these polishing techniques: Thanks to Mike McKenna's group in Lab 6 for polishing the prototype fibers using the teflon block technique. Thanks to Glenda Adkins, Eileen Hahn and Jack Upton of Technical Centers in Lab 7 at Fermilab for ice polishing these prototype fibers. Thanks to Alan Bross and Anna Pla for letting us borrow their single fiber polisher and to Carl Lindenmeyer for his work in designing the polisher, making the special collets required to polish the HP fiber and his general polishing expertise.

\section{References}

[1] T.Diehl et al., "Central Muon Upgrade Technical Design Report", DØ Internal Note 3365 .

[2] V.Abramov et al., "Forward Muon System for the D $\varnothing$ Detector Upgrade", NIM A419 (1998) 660-666.

[3] L.Sawyer et al., "Technical Design Report for the Upgrade of the ICD for DØ Run II", DØ Internal Note 2686 and FERMILAB-TM-2041, June 1997.

[4] Hewlett-Packard Co., 3000 Hanover Street, Palo Alto, California 94304. Ph. (650)8571501.

[5] P.Hanlet et al., "LED Pulser System for the DØ Muon Upgrade Scintillation Counters", DØ Internal Note 3563.

[6] E.Gallas and J.Li, "Polishing Optical Fibers for the DØ ICD in Run II ", DØ Internal Note 3390 and FERMILAB-TM-2062, November, 1998.

[7] For more information or a demonstration of the Fiberfin III, contact PM MFG. Service, Inc., Ph. (630) 553-6924, Fax (630) 553-9025, website www.PM MFG.com, email PMMFG1.aol.com. 